\title{
Is periareolar incision a suitable option for breast surgery? A mathematical comparison between periareolar and inframammary fold approaches
}

\author{
This article was published in the following Dove Press journal: \\ Open Access Surgery \\ 13 February 2017 \\ Number of times this article has been viewed
}

\section{Alessandro Borgognone' Giulio Gherardini ${ }^{2}$ Luigi Gliosci' Daniele D'Andria ${ }^{2}$}

'ASL ROMA 2, Division of Burn and Plastic Surgery, Sant'Eugenio Hospital - CTO Hospital, ${ }^{2}$ Private Practice, Rome, Italy
Correspondence: Alessandro Borgognone Division of Burn and Plastic Surgery, Sant'Eugenio Hospital - CTO Hospital, Piazzale Dell'Umanesimo, No 10, 00।44 Rome, Italy

Tel +390651002803

$\mathrm{Fax}+390651002906$

Email borgognone@gmail.com
Background: Several incisions have been proposed for the insertion of breast implants, but it is still debated which is the most conservative for the integrity of the prosthesis. There are no reports comparing periareolar and inframammary incisions on a mathematical basis. This study analyzed the efficacy of the two incisions through a detailed comparison based on increasing incision lengths to enable a more directed clinical choice.

Materials and methods: An 8-cm scale was plotted, representing either the linear section of an inframammary incision or the diameter of an ideal circumference; the corresponding hemi-circumference was calculated, and the advantage in length of the curvilinear section was determined.

Results: The data analysis confirmed a statistically significant increase of $36 \%$ in length when a curvilinear section was compared to an inframammary fold incision of the same length as the diameter of the related curvilinear section.

Conclusion: A periareolar incision may be a more effective technique to reduce the mechanical stress over cohesive gel breast implants during its insertion.

Keywords: periareolar incision, inframammary incision, comparison of mammary incisions, breast implant incisions

\section{Introduction}

Surgery involving breast implants is among the most common procedure performed by plastic surgeons in the US. In 2014, 284,254 augmentation mammoplasties and 8,455 reconstructive breast procedures with implant alone as well as 74,694 with tissue expander and implant were performed. ${ }^{1}$ Since the introduction of the last generation of shaped, silicone cohesive gel implants, the recommended incision site for the insertion of the implants and its length have been strongly debated. ${ }^{2}$

Beyond skin incision and its location, there are different and important factors potentially affecting the easiness of the implant insertion and the complications that might arise, they include, in a not exhaustive list, the implant surface texturing such as the "macrotexturing" of Natrelle ${ }^{\circledR}$ (Allergan, Inc, Irvine, CA, USA), the Siltex "microtexturing" of Mentor ${ }^{\circledR}$ (Mentor Corporation, Santa Barbara, CA, USA), and finally, the new nanotexturing of Motiva Implants ${ }^{\circledR}$ (Establishment Labs S.A., Alajuela, Costa Rica). ${ }^{3}$ 
Besides, it is noteworthy reporting the preoperative breast tissue and the volume of the implant selected as critical factors in addition to the former list, which are able to greatly influence the location of the skin incision that is more convenient.

The inframammary fold (IMF) incision is the most popular incision for breast augmentation; ${ }^{4}$ it has been shortened to as small as $1.7 \mathrm{~cm}$ to insert smooth, round inflatable implants, but even this size leaves visible scars, ${ }^{5}$ prompting the development of more precise methods; ${ }^{6}$ on the other hand, some authors maintain that the incision site is not of any concern. ${ }^{7,8}$

To better define this issue, it is widely agreed on that an appropriate incision length, regardless of the location, is needed to avoid damaging the architecture of the silicone core of the prosthesis. ${ }^{9}$ To try to take as much advantage as possible of the diameter of small areolae, a new modification of the nipple-areola complex (NAC) incision has been recently introduced..$^{10}$ Actually, Atiyeh et al, ${ }^{11}$ in 2002, already pioneered a new method of introducing inflatable as well as prefilled mammary implants through a very small intra-areoalar, infra-nipple incision with outstanding cosmetic results.

This confirms the advantages and the flexibility shown by the NAC site even at the more restricted area of the base of the nipple through which it is possible to perform a proper pocket and insert the more adequate mammary implant.

Once no significant differences are ascertained between women who undergo inframammary and periareolar approaches of mammaplasty, ${ }^{12}$ the incision length afforded by a curvilinear section and the choice of the most proper approach for inserting the anatomical form - stable implants - should be considered. To our knowledge, this is the first report of a mathematical comparison of linear IMF and periareolar access incisions.

\section{Materials and methods}

The data reported in this study are not drawn from the results of cadaveric or clinical studies; instead, they are based on the concept of biogeometry, which correlates mathematics with biological dynamics. ${ }^{12}$

We used a mathematical simulation model and plotted its related chart: the independent variable represents eight clinical simulations and the dependent variables correspond to the diameter of the areola or the linear IMF incision, the hemiperiareolar length, and the differences between them.

To determine the value of the hemiperiareolar variable, the formula $C=2 \times \pi r$ was applied for calculation of the circumference from the radius.
Table I Comparisons of the theoretical areola curvilinear diameter and the corresponding inframammary incision length

\begin{tabular}{lll}
\hline $\begin{array}{l}\text { Areola } Q \text { or IMF } \\
\text { incision } \mathbf{( c m})\end{array}$ & $\begin{array}{l}\text { Hemiperiareolar } \\
\text { incision } \mathbf{( c m})\end{array}$ & $\begin{array}{l}\text { Incision length } \\
\text { difference } \mathbf{( c m})\end{array}$ \\
\hline 1 & 1.57 & 0.57 \\
2 & 3.14 & 1.14 \\
3 & 4.71 & 1.71 \\
4 & 6.28 & 2.28 \\
5 & 7.85 & 2.85 \\
6 & 9.42 & 3.42 \\
7 & 10.99 & 3.99 \\
8 & 12.56 & 4.56 \\
Average & & \\
4.5 & 7.065 & 2.565 \\
& & $p<0.005$ \\
\hline
\end{tabular}

Abbreviations: $Q$, diameter of the periareolar incision; IMF, inframammary fold.

The dependent variables were increased by $1 \mathrm{~cm}$ from 1 to $12 \mathrm{~cm}$; we analyzed eight hypothetic cases comparing the independent variables (Table 1).

\section{Statistical analysis}

All analyses were performed using Microsoft Excel 2013 (Microsoft ${ }^{\circledR}$ Corporation, Redmond, WA, USA). The data were analyzed using descriptive summary statistics; a paired sample $t$-test was performed to determine whether differences existed in the progressive parametric values of the curvilinear sections and their corresponding diameters (linear section). The $95 \%$ confidence interval (CI) for the mean difference between the curvilinear and straight incisions was 1.40-3.74. The analysis was two-tailed, and statistical significance was determined at $p<0.005$.

\section{Results}

The intuitive observation (Figure 1) that the circumference is longer than its diameter and that the same applies to the hemi-circumference was mathematically confirmed.

Statistically, the paired $t$-test was performed to verify whether the curvilinear incision was effectively able to allow more room for insertion of a breast implant. The mean length increase $(M=2.525, \mathrm{SD}=1.40, \mathrm{n}=8)$ was significantly greater than 0 , with $t=5.20$ and two-tailed $p=0.0013$; this suggests that the periareolar incision provides greater length for breast implant insertion than a straight one. A periareolar incision provides $36 \%$ more length than the corresponding linear section (Figure 2). This relationship is expressed by the constant $\pi$. Thus, if it is assumed that the areolar diameter falls most often in the range of 3-6 cm, the hemiareola will be in the range of 


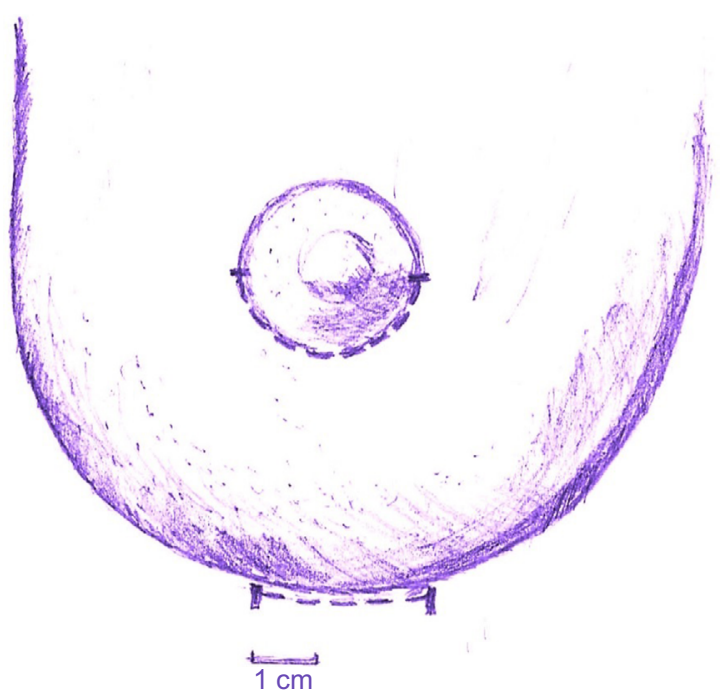

Figure I Visual comparison of the difference between a straight incision and a curvilinear incision with a diameter of the same length.

$4.71-9.42 \mathrm{~cm}$. The following are the steps and the results according to the formula:

$$
C=2 \times \pi r
$$

where $C$ is the areola and $r$ is half the areolar diameter. The simulation for an areolar diameter of $4 \mathrm{~cm}$ is:

$$
\begin{aligned}
& C=2 \times \pi \times 2, \\
& C=2 \times 3.14 \times 2, \\
& C=12.56 \mathrm{~cm} .
\end{aligned}
$$

In this case, an inferior hemiperiareolar incision would allow for an access of no less than $6.28 \mathrm{~cm}$.

\section{Discussion}

Recently, the pros and cons of implant characteristics and the choice of the most convenient incision for its placement have been disputed. This is true both for reconstructive and aesthetic surgeries.

In breast reconstructions with implants, the incision mostly practiced is in the mastectomy scar. In a series of 4,912 breast reconstructions with implants, $50.6 \%$ received Natrelle 410 FX (Allergan, Inc) and 82.6\% had the incision placed in the mastectomy scar. In this setting, the choice between IMF incision and periareolar incision is irrelevant. On the contrary, in breast augmentation, both result to be used respectively in $15.9 \%$ and $0.5 \%$ of cases. ${ }^{13}$ Some authors state that the location and its choice in this latter setting are meaningless, ${ }^{14}$ notwithstanding the IMF incision remains the most commonly used. ${ }^{15}$

This would be justified by the ease of performance, the supposedly low risk of implant contamination, biofilm formation, ${ }^{16}$ capsular contraction, ${ }^{17}$ and an overall lower complication rate. ${ }^{18}$

In fact, in 5,109 implantations for breast augmentation, periareolar incisions had a $6.9 \%$ incidence of capsular contracture compared with $4.5 \%$ for those placed through an inframammary incision; ${ }^{19}$ others found that transaxillary incisions produced the highest incidence of contracture $(6.4 \%)$, followed by periareolar $(2.4 \%)$ and inframammary $(0.5 \%)$ incisions. ${ }^{20}$

The first requirement acknowledged to prevent some fearful complications such as rotation, malposition, and asymmetry that are able to affect breast augmentation despite the site of insertion ${ }^{21}$ is the control of the pocket dimensions, which need be as tightly adherent as possible to the anatomic implant.

Therefore, the most proper skin incision will be that which allows the greatest control of the pocket with its boundaries

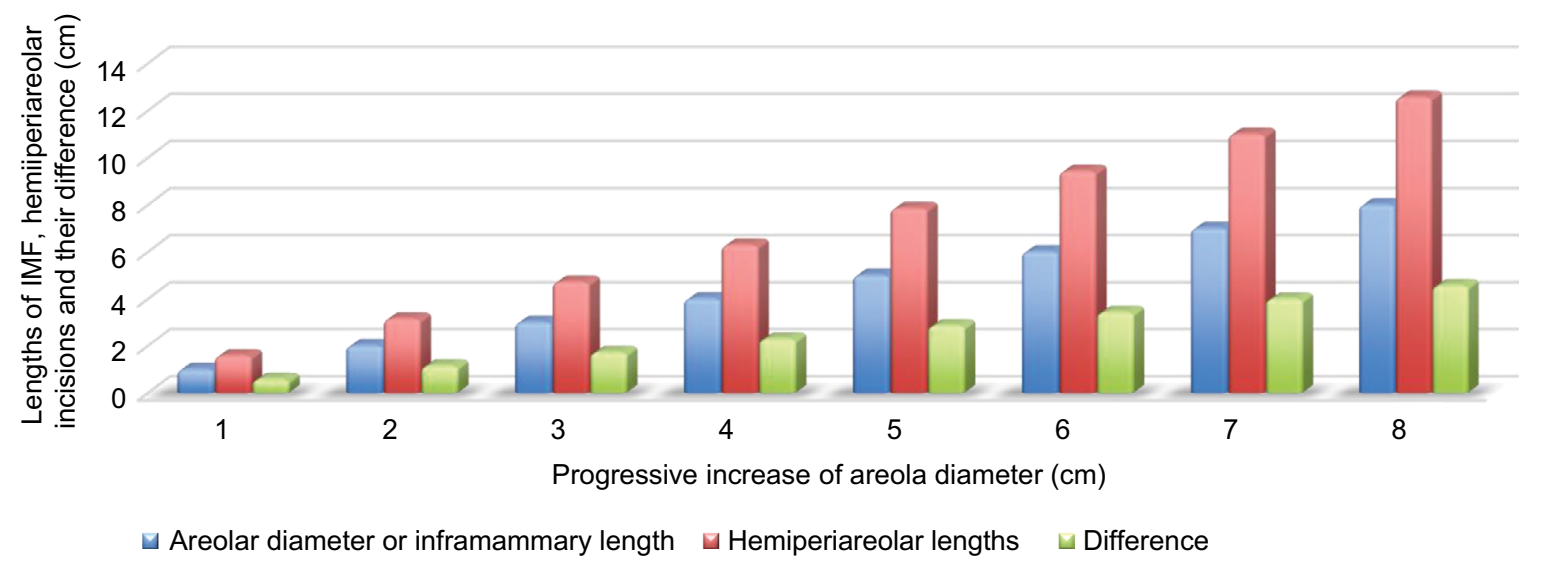

Figure 2 Comparison of a linear and a hemiperiareolar section: the straight incision and the diameter of the curvilinear section are of the same length. Abbreviation: IMF, inframammary fold. 
and an easy repositioning of the IMF; both incisions for this respect equally seem to share these advantages.

The argument that periareolar incision is associated with increased complications such as capsular contraction is commonplace but becomes debatable, if a correct and standardized protocol to keep the implant clean through the skin and the mammary gland is implemented..$^{22-24}$

To date, there are no reports in the literature of a diathesis favoring keloid formation. Even for racial factors, areolar and periareolar incisions are comparable to other adjacent skin areas such as the central sternum. ${ }^{25}$ Additionally, sensitivity of the NAC does not appear to be negatively affected by the periareolar incision. ${ }^{26}$

The appropriateness and convenience of a hemiperiareolar incision for breast augmentation revision have been well established, and no complications have been described to result from reusing the scar in this kind of revision surgery. ${ }^{27}$

Neither is the access at the NAC reported on as a potential risk factor for malposition or rotation of the anatomic implant due to the lack of control over the pocket nor are the difficulties increased for a resettling of the IMF, if need be.

Actually, it is just the case to highlight the widespread consensus on the use of the areola for the creation of the new IMF in the tuberous breast correction. ${ }^{28}$

As a matter of fact, the risk factors identified in a recent prospective study carried out on primary augmentations, revision augmentations, primary reconstructions, and revision reconstructions for high cohesive implants, Natrelle 410, have been just the following: a longer incision size for the primary augmentations and a capsulectomy performed at the time of implantation for the primary reconstructions. ${ }^{29}$

Furthermore, even if the comparison of the two incisions from a cosmetic point of view does not give any advantage to one of them, it remains the variable for the IMF incision, when the anatomical implant is used, to rightly foresee where, after the implantation, a section should properly fall in the new lowered IMF.

Unless reliable methods of measurements are introduced and widely applied such as that recently proposed by Atiyeh et $\mathrm{al}^{30}$ for a correct planning of the IMF, it is common experience that the more expanded lower pole of the breast due to the anatomical implant creates a consistent uplift of the IMF incision.

Thus, the inherent margin of error for the IMF incision, if not properly planned, could lead to a scar on the lower pole of the breast, which turns out to be more conspicuous than the areola incision equivalent.

With a reported "gel fracture" incidence of $6.3 \%$ in revision reconstruction patients, ${ }^{31}$ a shorter incision length is highly desirable, the more so because a $3-6 \mathrm{~cm}$ window is advised to prevent any cohesive silicone implant fracture. ${ }^{32}$

Mohmand and $\mathrm{Ahmad}^{33}$ reported an average periareolar incision length of $5.8 \mathrm{~cm}$ (range $4-7 \mathrm{~cm}$ ) in 32 breast augmentations with silicone implants, with $59.4 \%$ receiving an implant $>305 \mathrm{~g}$ (the largest being $435 \mathrm{~g}$ ).

Our study is focusing on the reasons the periareolar incision may be warranted.

It has been verified that any increment in diameter of $1 \mathrm{~cm}$ is related to an increase of $3.14 \mathrm{~cm}(\pi)$ in the associated circumference and of $1.57 \mathrm{~cm}$ (half $\pi=1.57$ ) in the hemicircumference, i.e., an $\sim 36 \%$ advantage over a linear IMF.

Since anatomically the mean areolar diameter range is $4-5.25 \mathrm{~cm}^{34,35}$ and recent algorithms define an areola of $3 \mathrm{~cm}$ diameter as small, a hemiperiareolar access of $4.71 \mathrm{~cm}$ might be of particular interest in this sort of patients, for the safe insertion of a silicone implant.

The strongest is the suggestion of an incision at least $5 \mathrm{~cm}$ long, lest the cohesive gel should fracture, the more the access through the areola is warranted - actually, it provides $36 \%$ more length and, thus, less structural stress on the implant.

Although it could be argued that the IMF incision is fixed whatever the areola diameter is and that it has the potential to be extended, the incision at the areola level is curvilinear and for its own nature, when straightened, actually already stretched.

The literature suggests that $66.7 \%$ of patients who undergo primary breast surgery and silicone implantation have submammary incisions $5-6.9 \mathrm{~cm}$ long; ${ }^{36}$ hence, it is proper to consider whether a hemiperiareolar incision could provide an easier corresponding access of $5-6.9 \mathrm{~cm}$ for areolae with a diameter of $3-4 \mathrm{~cm}$.

Arguably, for any incremental centimeter of a linear section, the progressive accessibility in the curvilinear approach improves as much as more than one-third; this is especially useful when a less traumatic insertion of a cohesive silicone implant is required.

Besides, our data confirmed that a curvilinear section is more suitable and is better camouflaged in small areolae, where hemiperiareolar access has on average an advantage of $36.31 \%$.

When the areolar diameter is $\geq 5 \mathrm{~cm}$, a periareolar section becomes less convenient because too large and the advantage for large areolae actually shrinks.

In fact, a periareolar incision of $7.85 \mathrm{~cm}$ corresponding to a diameter of $5 \mathrm{~cm}$ would be aesthetically less desirable than a corresponding $5 \mathrm{~cm}$ IMF incision (Figure 2).

These considerations may warrant further studies of the statistical distribution of areolar diameter in patients needing 
breast implants, enabling specific conclusions about the best incision concealment achievable for a given range of areolar dimensions. Finally, it would also be of interest to investigate the correlation between incision length and implant volume, i.e., the threshold below which an incision is unsuitable for a given volume of prosthesis.

Our study has potential limitations. First, the data collected from a theoretical analysis might not be entirely accurate and might not apply to a clinical setting; in fact, areolae almost never have a regular circumference, and the inferences drawn rely solely upon geometrical data.

Second, in theory, the choice suggested in this report for the most convenient access would rely upon just a single mathematical variable.

Third, the study does not take into account the central role of the viscoelastic properties of the skin; the stretched incision, for real, opens a "window" wider than the original incision length without consistent counteraction over the inserted structure. However, our simulations seem encouraging enough as much as to confirm the convenience for the periareolar approach in a selected range of areolae.

\section{Conclusion}

The present study mathematically confirms that by comparing the curvilinear and linear incisions, the periareolar one may result in less stress on a breast implant.

The periareolar approach provides an advantage of $36 \%$ over inframammary access and might be especially beneficial for small areolae.

To sum up, the evidence presented here could have, in a selected range of patients, positive implications, and we trust that these data might be of interest for future clinical trials.

\section{Acknowledgments}

We acknowledge the support of pharmaceutical and implant suppliers and all public bodies for their support and providing data for reproduction in the study. We would like to thank Editage for editing assistance. This study received no outside funding.

\section{Disclosure}

The authors report no conflicts of interest in this article.

\section{References}

1. American Society of Plastic Surgery. Plastic Surgery Statistics Report; 2014. Available from: http://www.plasticsurgery.org/Documents/newsresources/statistics/2014-statistics/plastic-surgery-statsitics-full-report. pdf. Accessed November 18, 2015.

2. Spear SL, Bulan EJ, Venturi ML. Breast augmentation. Plast Reconstr Surg. 2006;118:188S-196S.
3. Abramo AC, De Oliveira VR, Ledo-Silva MC, De Oliveira EL. How texture-inducing contraction vectors affect the fibrous capsule shrinkage around breasts implants? Aesthetic Plast Surg. 2010;34(5):555-560.

4. Reece EM, Ghavami A, Hoxworth RE, et al. Primary breast augmentation. Aesthet Surg J. 2009;29(2):116-121.

5. Fanous N, Tawilé C, Brousseau JV. Minimal inframammary incision for breast augmentation. Can J Plast Surg. 2008;16(1):14-17.

6. Bouwer LR, Tielemans HJP, van der Lei B. The Pythagorean theorem as a tool for preoperative planning of a concealed scar in augmentation mammaplasty with round implants. Plast Reconstr Surg. 2015;135(1): $110-112$.

7. Feng LJ, Amini SB. Analysis of risk factors associated with rupture of silicone gel breast implants. Plast Reconstr Surg. 1999;104(4):955-963.

8. Wiener TC. Comparison of breast augmentation incisions and common complications. Aesthetic Plast Surg. 2013;37(2):475-476.

9. Tebbetts J. Failure of a "highly cohesive implant": What does it really mean? Plast Reconstr Surg. 2009;124(1):323-325.

10. Han HH, Kim KK, Lee KH, et al. Transareolar-perinipple (areolar Omega) zigzag Incision for augmentation mammaplasty. Plast Reconstr Surg. 2015;135(3):517e-525e.

11. Atiyeh BS, Al-Amm CA, El-Musa KA. The transverse intra-areolar infra-nipple incision for augmentation mammaplasty. Aesthetic Plast Surg. 2002;26(2):151-155.

12. Tenta TL, Keyes GR. Biogeometry: the logic in the process of selection, sitting, design, construction and transfer of flaps. Clin Plast Surg. 1985; 12(3):423-452.

13. Cordeiro PG, McGuire P, Murphy DK. Natrelle 410 extra-full projection silicone breast implants: 2-year results from two prospective studies. Plast Reconstr Surg. 2015;136(4):638-646.

14. Stutman RL, Codner M, Mahoney A, Amei A. Comparison of breast augmentation incisions and common complications. Aesthetic Plast Surg. 2012;36(5):1096-1104.

15. Lista F, Ahmad J. Evidence-based medicine: augmentation mammaplasty. Plast Reconstr Surg. 2013;132(6):1684-1696.

16. Jacombs $\mathrm{A}$, Tahir $\mathrm{S}, \mathrm{Hu} \mathrm{H}$, et al. In vitro and in vivo investigation of the influence of implant surface on the formation of bacterial biofilm in mammary implants. Plast Reconstr Surg. 2014;133(4):471e-480e.

17. Namnoum JD, Largent J, Kaplan HM, Oefelein MG, Brown MH. Primary breast augmentation clinical trial outcomes stratified by surgical incision, anatomical placement and implant device type. J Plast Reconstr Aesthet Surg. 2013;66(9):1165-1172.

18. Somogyi RB, Brown MH. Outcomes in primary breast augmentation: a single surgeon's review of 1539 consecutive cases. Plast Reconstr Surg. 2015;135(1):87-97.

19. Stevens WG, Nahabedian MY, Calobrace MB, et al. Risk factor analysis for capsular contracture: a 5-year Sientra study analysis using round, smooth, and textured implants for breast augmentation. Plast Reconstr Surg. 2013;132(5):1115-1123.

20. Jacobson JM, Gatti ME, Schaffner AD, Hill LM, Spear SL. Effect of incision choice on outcomes in primary breast augmentation. Aesthet Surg J. 2012;32(4):456-462.

21. Jewell M. Form stable silicone gel breast implants. Clin Plast Surg. 2009;36(1):75-89.

22. Shestak KC, Askari M. A simple barrier drape for breast implant placement. Plast Reconstr Surg. 2006;117(6):1722-1723.

23. Pfeiffer P, Jørgensen S, Kristiansen TB, Jørgensen A, Hölmich LR. Protective effect of topical antibiotics in breast augmentation. Plast Reconstr Surg. 2009;124(2):629-634.

24. Zambacos GJ, Nguyen D, Morris RJ. Effect of povidone iodine on silicone gel breast implants in vitro: implications for clinical practice. Plast Reconstr Surg. 2004;114(3):706-710.

25. Rockwell WB, Cohen IK, Ehrlich HP. Keloids and hypertrophic scars: a comprehensive review. Plast Reconstr Surg. 1989;84(5):827-837.

26. Mofid MM, Klatsky SA, Singh NK, Nahabedian MY. Nipple-areola complex sensitivity after primary breast augmentation: a comparison of periareolar and inframammary incision approaches. Plast Reconstr Surg. 2006;117(6):1694-1698. 
27. Luo SK, Chen GP, Sun ZS, Cheng NX. Our strategy in complication management of augmentation mammaplasty with polyacrylamide hydrogel injection in 235 patients. J Plast Reconstr Aesthet Surg. 2011;64(6):731-737.

28. Mandrekas AD, Zambacos GJ. Tuberous breast deformity: classification and treatment. Plast Reconstr Surg. 2015;136(2):269e.

29. McGuire P, Reisman NR, Murphy DK. Risk factor analysis for capsular contracture, malposition, and late seroma in subjects receiving Natrelle 410 form-stable silicone breast implants. Plast Reconstr Surg. 2017;139(1):1-9.

30. Atiyeh BS, Dibo SA, Nader M, Papazian NJ. Preoperative assessment tool for the planning of inframammary incision and implant profile in breast augmentation. Aesthetic Plast Surg. 2014;38(5):878-886.

31. Direction for Use - Allergan NATRELLE 410 Highly Cohesive Anatomically Shaped Silicone-Filled Breast Implants. Available from: www.allergan.com/miscellaneous-pages/allergan-pdf-files/ldoc03824_410_aug. Accessed November 18, 2015.
32. Mentor Product Insert Data Sheet; July 2009. Available from: http:// www.fda.gov/downloads/MedicalDevices/ProductsandMedicalProcedures/ImplantsandProsthetics/BreastImplants/UCM245623.pdf. Accessed November 18, 2015.

33. Mohmand MH, Ahmad M. Periareolar extra-glandular breast augmentation. World J Plast Surg. 2013;2(2):93-98.

34. Sanuki J, Fukuma E, Uchida Y. Morphologic study of nipple-areola complex in 600 breasts. Aesthetic Plast Surg. 2009;33(3):295-297.

35. Pérez-Guisado J, Rodríguez-Mérida C, Rioja LF. Areola size and jugulum nipple distance after bilateral mastectomy and breast reconstruction. Eplasty. 2013;13:e56.

36. Picha GJ, Singh N, Murphy DK. Natrelle silicone breast implant follow-up study: demographics, lifestyle, and surgical characteristics of more than 5000 reconstruction subjects. Plast Reconstr Surg. 2015; 3(8):e489.
Open Access Surgery

\section{Publish your work in this journal}

Open Access Surgery is an international, peer-reviewed, open access journal that focuses on all aspects of surgical procedures and interventions. Patient care around the peri-operative period and patient outcomes post surgery are key topics for the journal. All grades of surgery from minor cosmetic interventions to major surgical procedures are covered. Novel techniques and the
Dovepress

utilization of new instruments and materials, including implants and prostheses that optimize outcomes constitute major areas of interest. The manuscrip management system is completely online and includes a very quick and fair peer-review system, which is all easy to use. Visit http://www.dovepress.com testimonials.php to read real quotes from published authors. 